\title{
Serotonergic Degeneration in Parkinson's Disease: What Is the Link With the Age of Onset?
}

\author{
Antonella Macerollo
}

Keywords: Serotonergic network, Neurodegeneration, Parkinson's Disease

doi:10.1017/cjn.2020.11

Can J Neurol Sci. 2020; 47: 287-288

It is well known that Parkinson's disease (PD) is caused by degeneration of nigrostriatal dopaminergic neurons as well as several neurotransmitter pathways, especially the serotonergic network. However, the significance and the contribution of these neuronal networks to various aspects of PD remain enigmatic. In turn, the involvement of different neuronal networks leads to a large number of motor and non-motor symptoms characterizing this disease. Serotonergic dysfunction has been related to a variety of motor (e.g., dyskinesia) and non-motor symptoms (e.g., sleep difficulties, fatigue, and depression).

Neurophysiological studies using different techniques and basic science studies have investigated the role of the serotonergic degeneration in PD. The involvement of serotonergic neurons in the pathophysiology of this movement disorder has been showed in animal studies. ${ }^{1}$ Much of what is currently known about the role of this network in the pathophysiology of PD is derived from research combining neurophysiological techniques with behavioral measures of movement. In addition, behavioral and neurophysiology studies in clinical populations with the basal ganglia affected such as PD have been critical in advancing our understanding of the serotonergic system.

Determining how serotonergic neurons contribute to specific aspects of motor and non-motor symptoms of PD will help in understanding how the serotonergic system contributes to the clinical phenotype of PD. Both animal and human research is critical to developing a thorough understanding of this topic. Furthermore, neurophysiological methods that consider the sensory-motor and connected biological system as a whole, including auditory evoked potentials, are integral to advancing knowledge on this topic.

In this regard, there were two unanswered questions regarding the serotonergic system in PD. Does the age of PD onset influence the involvement of serotonergic system? In other words, is there any difference between early-onset and late-onset $\mathrm{PD}$ in serotonergic neurodegeneration, which might explain differences in their clinical features? Additionally, what is the impact of dopaminergic medications on this network? Understanding these two aspects is important in developing curative therapies as well as improving symptomatic management of PD.

In this issue, Park and colleagues applied a neurophysiology protocol based on loudness dependence of the auditory evoked potentials (LDAEP) to investigate the central serotonergic neurotrasmission since it is well known that serotonergic innervation is particularly high in the auditory cortex. ${ }^{2}$ Overall, these authors aimed to test the two research questions listed above. ${ }^{2}$ LDAEP was measured using N100 as well as P200 amplitude elicited by auditory stimuli as previously evaluated in PD by Beucke et al. ${ }^{3}$

The study was performed on 30 mediation naïve PD patients divided into two groups: early- ( $<69$ years) and late-onset PD ( $>70$ years). This innovative study found that the absolute values of pre-treatment N1/P2 LDAEP and post-treatment N1 LDAEP (12 weeks after dopaminergic treatment) were lower in the early-onset group than in the late-onset group, suggesting that central serotonergic activity was more preserved in the early-onset compared with the late-onset group. Moreover, the authors found that dopaminergic medications normalized the N1 LDAEP causing significant amplitude decrease after 12 weeks of treatment.

These findings might explain some phenotypic differences related to the age of onset in PD. In particular, levodopa-induced dyskinesias have recently been showed to be associated with loss of balance between serotonin and dopamine neurotransmission with a longer preserved serotonin activity in the presence of earlier dopaminergic degeneration. ${ }^{4}$ Indeed, an enhanced striatal serotonergic innervation is a proposed compensatory mechanism for the dopaminergic deficit.

Consequentially, these results should lead to research studies to develop new treatments for dyskinesia targeting specifically on the serotonin activity. Moreover, LDAEP is a promising neurophysiological biomarker of serotonin neurotransmission which may be used to identify patients who may be helped by new therapies. However, the timing of serotonergic system degeneration in the course of PD is still under debate.

A recent study on the status of striatal serotonergic innervations in MPTP (1-methyl-4-phenyl-1,2,3,6-tetrahydropyridine)intoxicated monkeys at different stages of experimental

From the Walton Centre for Neurology and Neurosurgery, Department of Neurology, Liverpool, UK; Faculty of Health and Life Sciences, University of Liverpool, Liverpool, UK

Received December 26, 2019. Date of Acceptance January 2, 2020. Correspondence to: Antonella Macerollo, Walton Centre for Neurology and Neurosurgery, Department of Neurology, Liverpool, UK; Faculty of Health and Life Sciences, University of Liverpool, Liverpool, UK. Email: a.macerollo@liverpool.ac.uk 
parkinsonism did not find any changes in serotonin positive axonal density in the caudate or putamen, in either the presymptomatic or symptomatic stage of parkinsonism, not supporting a compensatory role for the striatal serotonergic system. ${ }^{6}$ However, it is well known that the serotonergic dysfunction in PD involves not only the basal ganglia but also other regions, such as the raphe nuclei. In this regard, Pasquini et al have recently showed that about 4 years after diagnosis, raphe serotonin transporter availability was significantly reduced in more than one-third of PD patients without correlation to non-motor symptoms but with a correlation to tremor severity. ${ }^{7,8}$

Park and colleagues tried to explore the role played by dopaminergic agents on the serotonin system. The study showed that dopaminergic treatments change the pathologically high LDAEP. Consequentially, dopaminergic therapies might be optimized to improve the serotonergic activity by measuring changes of LDAEP as a disease biomarker. Moreover, measuring serotonergic activity through LDAEP may be helpful to avoid damage of serotonergic neurons due to high pharmacological dopaminergic stimulation.

While the findings of Park et al are intriguing, it would be interesting to perform a similar study with an age-matched control group to rule out a potential role of age in the serotonin system degeneration that might explain the differences in serotonergic activity between the early- and late-onset PD patients. Moreover, there is still a lack of consensus in the age definition of early- and late-onset PD. This effect of age on the serotonergic system and the arbitrary choice of the age to identify early-onset and late-onset PD may underlie the contrasting results among different studies. However, it is likely that further progresses in animal studies, neuroimaging techniques, basal ganglia connectivity, and computational processing will find their way to clinical practice and open up a plethora of research possibilities in the coming decade.

\section{Disclosures}

The author has no conflicts of interest to declare.

\section{REFERENCES}

1. Halliday GM, Blumbergs PC, Cotton RG, Blessing WW, Geffen LB. Loss of brainstem serotonin- and substance P-containing neurons in Parkinson's disease. Brain Res. 1990;510:104-7.

2. Park HK, Lee JJ, Park YM. Preserved serotonergic activity in earlyonset Parkinson's disease. Can J Neurol Sci. 2019:1-6. doi: 10.1017/cjn.2019.322. [Epub ahead of print].

3. Beucke JC, Uhl I, Plotkin M, et al. Serotonergic neurotransmission in early Parkinson's disease: a pilot study to assess implications for depression in this disorder. World J Biol Psychiatry. 2010; $11: 781-7$

4. Pagano G, Niccolini F, Politis M. The serotonergic system in Parkinson's patients with dyskinesia: evidence from imaging studies. J. Neural Transm. 2018;125:1217-23.

5. Boulet S, Mounayar S, Poupard A, et al. Behavioral recovery in MPTP-treated monkeys: neurochemical mechanisms studied by intrastriatal microdialysis. J. Neurosci. 2008;28:9575-84.

6. Jiménez-Sánchez L, Blesa J, Del Rey NL, Monje MHG, Obeso JA, Cavada C. Serotonergic innervation of the striatum in a nonhuman primate model of Parkinson's disease. Neuropharmacology. 2019:107806. [Epub ahead of print].

7. Pasquini J, Ceravolo R, Brooks DJ, Bonuccelli U, Pavese N. Progressive loss of raphe nuclei serotonin transporter in early Parkinson's disease: a longitudinal 123I-FP-CIT SPECT study. Parkinsonism Relat Disord. 2019. [Epub ahead of print]

8. Jankovic J. Parkinson's disease tremors and serotonin. Brain. 2018;141(3):624-6. 